\title{
The Effect of Recombinant Human Bone Morphogenetic Protein-7 on the Osteoblast-like Cells Cultured on Implant
}

\author{
Mahmoud Ibrahim Nassar ${ }^{1 *}$, A. Ahmed ${ }^{2}$, A. Dina ${ }^{3}, A$. Abdullah $^{2}$ \\ ${ }^{1}$ Department of Oral Medicine, Oral Diagnosis and Periodontology, Faculty of Dentistry, Aswan University, Tingar, Egypt; \\ ${ }^{2}$ Department of Oral Medicine, Oral Diagnosis and Periodontology, Faculty of Dentistry, Minia University, Minia, Egypt; \\ ${ }^{3}$ Department of Medical Biochemistry and Molecular Biology, Faculty of Medicine, Cairo University, Cairo, Egypt
}

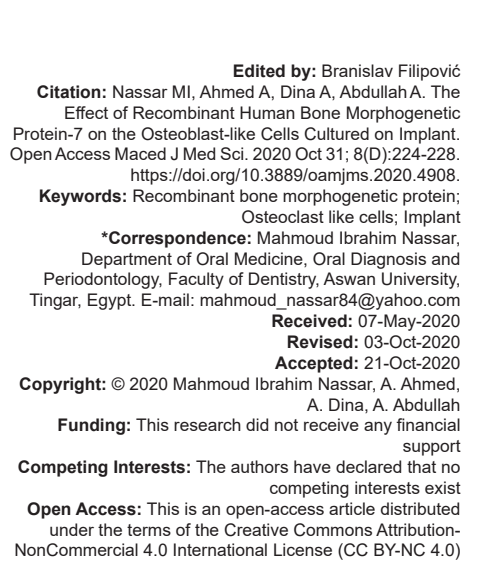

Abstract

AIM: The aim of the study was to study the effect of recombinant human bone morphogenetic protein-7 (rhBMP-7) on the osteoblast-like cells cultured on implant.

MATERIALS AND METHODS: The osteoblast-like osteo-1 cell line was used in this experiment and derived from the parietal bone tissue of newborn albino rats. The cells were incubated in a humid atmosphere of $95 \%$ air and $5 \%$ carbon dioxide at $37^{\circ} \mathrm{C}$. The medium was changed every 2 days. Four groups were conducted as follows osteoclastlike cell (machined), implanted osteoclast-like cell on titanium (Ti) (modified), osteoclast-like cell supplemented with BMP-7 (Machined + BMP-7), and implanted osteoclast-like cell on Ti and supplemented with BMP-7 (Modified + BMP-7)

RESULTS: Cell proliferation was influenced by rhBMP-7, as demonstrated by a significant increase in collagen content after 7 and 21 days of culture $(p=0.005)$ and a significant increase in alkaline phosphatase (ALP) activity after 7 days $(p<0.001)$. The addition of rhBMP-7 influenced ALP activity, and a significant increase was observed after 21 days $(p<0.001)$.

CONCLUSION: Within the limitations of the study, we concluded that the presence of rhBMP-7 did not influence cell adhesion to chemically modified Ti surfaces but provided an additional stimulus during the differentiation of rat osteo-1 cells cultured on this type of surface.

\section{Introduction}

Oral or endosseous implants (also known as dental implants) have been used to replace missing teeth for more than half a century. Considering it to be an important contribution to dentistry as they have altered the way by which missing teeth are replaced with a high success rate. This success depends on the ability of the implant material to integrate with the surrounding tissue. However, this fusion is affected by several factors, such as implant material, bone quality and quantity, and the implant loading condition [1].

Several lines of evidence indicated that an accumulation of bacteria on the implant surface plays an important role in the etiology of peri-implantitis - an inflammatory condition affecting the tissues around osseointegrated implants, leading to loss of supporting bone. The main aim in the treatment of peri-implantitis is to arrest the progression of the disease and at the same time to keep the dental implant in function, solving the inflammatory signs of bleeding and pain. Periimplant bony defects around dental implants can be treated with either non-surgical or surgical (resective or regenerative) techniques. Bone tissue regeneration is the objective therapeutic option in selected peri-implant bony defects of functioning implants if appropriate surgical techniques are utilized and the etiologic cause is fully eradicated [2].

The regenerative approach attempts to regenerate bone around peri-implantitis sites. The materials used are bone grafts, with or without membranes, or membrane alone. Biologic agents, such as growth factors or bone morphogenetic proteins (BMP), can be considered [3].

Researches have been conducted for over the years finding the treatment procedures for the regeneration of a lost or injured part so that form and function of lost structures can be regained. This regenerative field holds the promise of engineering damaged tissues with the help of various growth factors, including bone morphogenetic proteins (BMPs), by stimulating the body's own repair mechanisms [3]. BMPs are a group of signaling molecules that belong to the transforming growth factor- $\beta$ superfamily of proteins. Originally identified for their ability to induce bone formation [4], BMP signaling plays a role in the regulation of adipose tissue, with different members 
of the family playing different and sometimes opposite roles. Recombinant human BMPs (rhBMPs) are used in orthopedic applications such as spinal fusions, nonunions, and oral surgery. rhBMP-2 and rhBMP-7 are food and drug administration approved for some uses. RhBMP-2 causes more overgrown bone than any other BMPs and is widely used off-label [5].

\section{Materials and Methods}

This work was performed at the unit of the biochemistry and molecular biology, medical biochemistry, and molecular biology Department, Faculty of Medicine, Cairo University, Egypt.

\section{in the following}

The steps of this study can be summarized

i. Osteoclast had been isolated and grown in a sterile medium at $37^{\circ} \mathrm{C}$ and maintained in vitro under standard culture conditions (Table 1).

Table 1: Preparation of cell growth medium

\begin{tabular}{ll}
\hline Cell Growth medium preparation & $\mathrm{ml}$ \\
\hline RPMl-1640W/stable glutamine sterile filtered (bio west, Nampa, catn L0498-500): & $450 \mathrm{ml}$ \\
$50 \mathrm{ml}$ was removed from 500 ml bottle, then the other constituents were added & \\
$10 \%$ FBS (PAA, Pasching Austria, Cat nA11-151) & $50 \mathrm{ml}$ \\
$100 \mathrm{U}$ penicillin/0.1 mg/ml streptomycin (Lonza, Verviers, Belgium, CatN & $5 \mathrm{ml}$ \\
DE17-602E) & \\
\hline
\end{tabular}

ii. Cultured cells were treated by BMP-7 with and without commercially available titanium (Ti) (ASTM F67). Ti ASTM-F67 specification was unalloyed $\mathrm{Ti}$ for surgical implant applications (UNS R50250, UNS R50400, UNS R50550, UNS R50700)

iii. BMP-7 (Sigma-Aldrich Chemical Co., St. Louis, Mo, U.S.A., product number: B1434, MDL: MFCD03702812) was supplemented for our research groups. Our research groups were shown in (Table 2)

Table 2: Cell culture groups

\begin{tabular}{ll}
\hline Group number & Culture description \\
\hline Group 1 & OLC (Machined) were cultured for 1 and 3 weeks \\
Group 2 & Implanted OLC on Ti (Modified) were cultured for 1 and 3 weeks \\
Group 3 & OLC supplemented with BMP-7 (Machined + BMP-7) were cultured for \\
Group 4 & $\begin{array}{l}\text { 1 and 3 weeks } \\
\text { Implanted OLC on Ti and supplemented with BMP-7 (Modified + BMP- } \\
\text { 7) were cultured for 1 and 3 weeks }\end{array}$ \\
\hline Ti: Titanium, BMP-7: Bone morphogenetic protein-7
\end{tabular}

iv. Cell proliferation assay performed to assess the cell viability of control and BMP-7 treated cells. Measurement of cell viability and proliferation comprises the underlying basis for numerous in vitro assays directed toward the quantitation of a cell population's response to external factors (different concentration of metformin). MTT reagent, supplied ready for use, was obtained from (Biospes, China, Cat n\#BAR1005-1) v. Alkaline phosphatase (ALP) activity was assessed

vi. Collagen Type I gene expression by real-time polymerase chain reaction was assessed, the kit was provided by Bioline, a median life science company, UK (SensiFAST TM SYBR ${ }^{\circledR}$ Hi-ROX One-Step Kit, Catalog no.PI-50217 V)

vii. Comet assay was evaluated, the comet assay was conducted under alkaline conditions with some modifications, basically as described by Singh et al. (1988) [6].

\section{Results}

\section{Cell proliferation}

Cell proliferation in mod and mod + BMP7 groups compared to machined group ( $p<0.001$ ), but no significant difference in cell proliferation between machined + BMP7 and machined group ( $p=0.6$ ). Significant decrease in cell proliferation in machined + BMP7 group compared to mod group and mod + BMP7 group $(p<0.001)$. Mod + BMP7 group shows the highest significant increase in cell proliferation.

\section{Collagen gene expression}

\section{After 1 week}

Significant increase in collagen gene expression in mod and mod +BMP7 groups compared to machined group ( $p=0.002,<0.001$, respectively), but no significant difference in collagen gene expression between machined +BMP7 and machined group ( $p=0.58)$. Significant increase in collagen gene expression in mod +BMP7 groups compared to machined +BMP7 group $(p=0.005)$.

\section{After 3 weeks}

Significant increase in collagen gene expression in mod and mod + BMP7 groups compared to machined group ( $p<0.001$ ), but no significant difference in collagen gene expression between machined + BMP7 and machined group ( $p=0.37$ ) Significant increase in collagen gene expression in mod + BMP7 groups compared to machined + BMP7 group $(p<0.001)$.

\section{ALP activity \\ Comet assay}

In 1 week duration, significant decrease in ////// in machined + BMP7 and mod + BMP7 compared to machined group ( $p=0.01,0.003)$, respectively, 
but no significant difference between mod group and machined group $(p=0.4)$. Significant decrease in $/ / / / / / /$ in machined + BMP7 and mod + BMP7 compared to mod group ( $p<0.001)$, no significant differences between machined + BMP7 and mod + BMP7 ( $p=0.98)$ while in 3-week duration we revealed that significant decrease in /////// in machined + BMP7 and mod + BMP7 compared to machined group ( $p$ $<0.001)$, but no significant difference between mod group and machined group $(p=0.1)$. Significant decrease in /////// in machined + BMP7 and mod + BMP7 compared to mod group $(p<0.001)$ and no significant differences between machined + BMP7 and $\bmod +\operatorname{BMP} 7(p=0.4)$.

\section{Discussion}

BMPs are multi-functional growth factors that belong to the transforming growth factorbeta superfamily. The roles of BMPs in embryonic development and cellular functions in postnatal and adult animals have been extensively studied in recent years. In vertebrates, BMP genes are expressed in many embryonic organs and tissues, including those in which reciprocal interactions between epithelial and mesenchymal cells are important for morphogenesis and differentiation [7].

Current advances in biomaterial science have led to the discovery of new materials for dental use and have broadened their use in preventive, restorative, and regenerative treatments. A wide variety of these materials ranging from dental cements, resins, metals, and alloys to ceramic materials are used in dentistry. Metals and alloys commonly used in dentistry include Ti and their alloys such as nickel-titanium (NiTi), stainless steel, cobalt-chrome alloys, nickel-chrome, gold-based alloys, or dental amalgam [6].

Polymers play a major role in different aspects of dentistry, such as preventive, restorative, and regenerative therapies. The use of polymeric materials and polymeric films rather than traditional materials (such as dental amalgam and cements) used in dentistry is becoming more common due to their physical and mechanical properties and biological properties. Moreover, these materials can be used for dentin regeneration or as advanced drug delivery systems. The osseointegration of $\mathrm{cpTi} / \mathrm{Ti}$ alloy implants used for dental, craniofacial, and orthopedic purposes is related to their composition and surface roughness. Rough surfaced implants increase osseointegration and biomechanical stability. Implant surface treatment methods include $\mathrm{Ti}$ plasma-spraying, grit-blasting, acid etching, iodization, and calcium phosphate coatings. A favorable environment is necessary for implant osseointegration. The reduced oxygen concentration from the poor vasculature at the implant surface interface promotes a buildup of host-cellrelated electrons as free radicals and proton acid that can encourage infection and inflammation, causing implant failure. To provide a favorable environment for osseointegration and overcoming the problems associated with $\mathrm{Ti}$ implants, surface modifications can be performed using high-strength fiber-reinforced and complex fillers/additives, including hydroxyapatite or antimicrobial incorporation through thermoset polymers, which cure at low temperatures. The polymer/carbon-fiber-reinforced composite produced successful osseointegration. Thermoset polymer matrix and carbon fibers generate covalent bonds providing strong bone structure support with excellent osseointegration [6].

A bioactive material can interact with the biological environment to elicit a specific biological response, such as the formation of a hydroxyapatite layer with a bond forming between the tissue and material. Bone and teeth, enamel and dentin, consists mainly of mineralized hard tissue in the form of hydroxyapatite, a crystalline calcium phosphate, Ca10 (PO4)6(OH) 2. In contrast, bio-inert materials do not elicit any specific responses or interact with the biological environment. However, they can result in a foreign-body reaction and the formation of a fibrous capsule. The fibrous capsule may result in micro-movements and eventual failure of the prosthesis. Bioactive materials may be osteoconductive or osteoinductive. The most bioactive glass (BAG) has a superior surface area with a higher dissolution rate and thus faster apatite formation. In addition, they have shown to increase the mechanical properties of such composite for natural bones and provide biomimetic nano-structuration enhancing cell adhesion [8].

To achieve adequate retention in bone, osseointegration, direct contact between the implant surface and bone tissue is needed. Ti-based alloys are the most widely used materials for dental implants. They are highly biocompatible and osteoconductive but are bio-inert. However, they provide attachment for the osteoprogenitor cells and osteoblasts and are undesirable for microorganisms. There are also reports of failed osseointegration of titanium-dental implants (Ti-DIs). The bio-inert nature of the Ti-Dls may benefit from the addition of BAG as BAG might help implants bond actively to the bone and provide antimicrobial protection and a reduction in total treatment time. Until today, no BAG coating for dental implants is commercialized for clinical use [8].

Consequently, the present study was carried out to provide further evidence on the effect of rhBMP-7 on the osteoblast-like cells cultured on the implant.

Our study was conducted and prepared on four different groups to asses the effect of recombinant bone morphogenic 7 on osteoblast cells cultured on titanium surfaces. 
Regarding cell proliferation in our study showed a significant increase in cell proliferation in mod and mod + BMP7 groups compared to the machined group but no significant difference in cell proliferation between machined + BMP7 and machined group. Significant decrease in cell proliferation in machined + BMP7 group compared to mod group and mod + BMP7 group. Mod + BMP7 group shows the highest significant increase in cell proliferation compared to all other groups, this was slightly different than what discovered by Togashi et al. 2009 that found no significant differences in the cell viability or the cell count between the different surfaces tested, irrespective of the presence or absence of rhBMP-7, his studied was agreed by Anselme and Bigerelle 2006 (83), although they have found a significant increase in total protein content in machined BMP7 group, they concluded that in all groups, the cells had reached an adequate level of total protein for the onset of the differentiation process at 14 days [9], [10].

The addition of rhBMP-7 to the culture medium did not seem to have contributed to the total protein content of osteo- 1 cells. A study by Tsuda et al. 2014 stated that the addition of rhBMP-7 did not influence cell adhesion, although it seems to be more involved in the process of cell differentiation [10].

Results in our study for collagen expression revealed that significant increase in collagen gene expression in mod and mod + BMP7 groups compared to the machined group but no significant difference in collagen gene expression between machined + BMP7 and machined group, while a significant increase in collagen gene expression in mod + BMP7 groups compared to machined + BMP7 group. It was also discovered that no significant difference in collagen gene expression in the machined group between 1- and 3-week duration while no significant difference in collagen gene expression in mod group between 1- and 3-week duration, also no significant difference in collagen gene expression in machined + BMP7 group between 1- and 3-week duration, while there was significant increase in collagen gene expression in mod + BMP7 3 weeks group compared to 1 -week duration. These results were equivalent with the findings of Martinovic et al. 2006 and Togashi et al. 2009 stating that the collagen matrix was already established after 7 days, adding to that the studies of Cooper et al. 2006 and Cirano et al. 2014 that stated that rhBMP-7 induced a numerical increase in collagen content after 7 days with no differences between groups were observed after 14 days, indicating that the collagen matrix is ready for mineralization after 7 days [11], [12].

In our study, ALP enzyme activity in 3-week duration showed that significant increase in ALP activity in machined + BMP7 and mod + BMP7 compared to machined group but no significant difference between mod group and machined group, on the other hand, activity in 4-week duration showed a significant increase in ALP activity in mod, machined + BMP7 and mod + BMP7 compared to machined group, respectively, but there was a significant increase in ALP activity in machined + BMP7 and mod + BMP7 compared to mod group. Our results regarding ALP enzyme activity were correlated with that of Togashi et al. 2009, where they found that the highest ALP activity levels at 14 days for the machined SLA mod and BMP-7 machined groups (Figure 1). The addition of rhBMP-7 had no effect on the amplification of cell differentiation or the resulting mineralization promoted by Osteo-1 cells grown on rough and chemically modified SLA surfaces. The results showed that the concentration of rhBMP-7 of $20 \mathrm{ng} / \mathrm{ml}$, in combination with Ti disks, did not increase ALP activity or the formation of calcified nodules. Martinovic et al. 2006 also observed no effect of BMP-7 on ALP activity during the stage of cell differentiation, they stated that Although there is no explanation for this

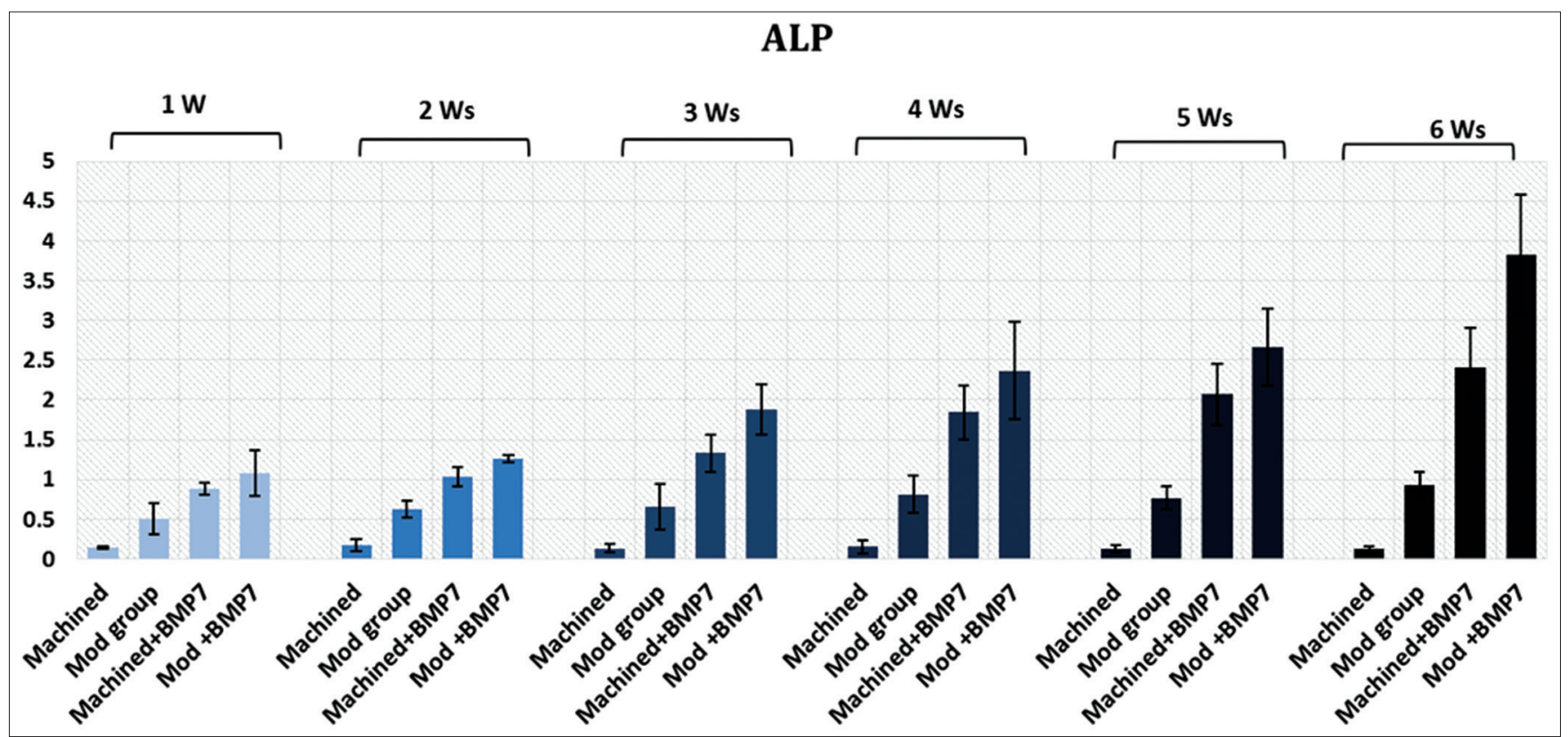

Figure 1: Our results for four groups compared along 6 successive weeks 
fact, it may be hypothesized that the presence of BMP-7 in the culture medium did not potentiate the response of cells grown on rough and chemically modified surfaces, because the molecular mechanisms, signaling molecules, ligands, and receptors involved in the differentiation of osteoblasts mediated by rough surfaces may have failed to interact because of their specificity or because of competition with osteoblasts mediated by BMP-7 through shared pathways. On the other hand, another hypothesis regarding cell differentiation may be suggested. The lack of observation of a significant difference in the BMP-7/ SLAmod group between 7 and 14 days may indicate that AP production had already reached a level at 7 days that favored the possible formation of a mineralized matrix, indicating earlier cell differentiation than that observed in the other groups. In another study by Chaudhari et al. 2004 reported that the same concentration of rhBMP-7 is necessary to produce a maximum effect on the stimulation of ALP activity and collagen synthesis, suggesting that the induction of ALP is directly or indirectly related to the synthesis or accumulation of collagen matrix. Treatment with rhBMP-2 and rhBMP-7 influenced ALP activity, and a significant reduction was observed after 21 days of culture and a non-significant decrease after 14 days. This reduction in ALP production suggests that the activity of this enzyme, an early differentiation marker, is reaching its plateau at 14 days [9], [10], [11], [12].

\section{Conclusion}

Within the limitations of the study, we concluded that the presence of rhBMP-7 did not influence cell adhesion to chemically modified titanium surfaces but provided an additional stimulus during the differentiation of rat osteo- 1 cells cultured on this type of surface.

\section{References}

1. Sharma A, Sharma H. Bone morphogenetic proteins: An overview. Ann Appl Biosci. 2017;4(2):R35-7. https://doi. org/10.21276/aabs.1336

2. Daugela $P$, Cicciù $M$, Saulacic $N$. Surgical regenerative treatments for peri-implantitis: Meta-analysis of recent findings in a systematic literature review. J Oral Maxillofac Res. 2016;7(3):e15. https://doi.org/10.5037/jomr.2016.7315 PMid:27833740

3. Rokaya D, Srimaneepong V, Wisitrasameewon W, Humagain M, Thunyakitpisal P. Peri-implantitis update: Risk indicators, diagnosis, and treatment. Eur J Dent. 2020;14(4):672-82. https://doi.org/10.1055/s-0040-1715779

PMid:32882741

4. Urist MR, Strates BS. Bone morphogenetic protein. J Dent Res. 1971;50(6):1392-406. https://doi.org/10.1177/00220345710500 060601

PMid:4943222

5. Uwagie-Ero EA, kene RO, Chilaka FC. Bone morphogenetic proteins an update and review. Trop J Nat Prod Res. 2017;1(1):1 11. https://doi.org/10.26538/tjnpr/v1i1.2

6. Rokaya D, Srimaneepong V, Sapkota J, Qin J, Siraleartmukul K, Siriwongrungson V. Polymeric materials and films in dentistry: An overview. J Adv Res. 2018;14:25-34. https://doi.org/10.1016/j. jare.2018.05.001

PMid: 30364755

7. Schnee CL, Weil RJ. Analysis of harvest morbidity and radiographic outcome using autograft for anterior cervical fusion. Spine (Phila Pa 1976). 1997;22(19):2222-7. https://doi. org/10.1097/00007632-199710010-00005

PMid: 9346142

8. Skallevold HE, Rokaya D, Khurshid Z, Zafar MS. Bioactive glass applications in dentistry. Int J Mol Sci. 2019;20(23):5960. https:// doi.org/10.3390/ijms20235960

PMid: 31783484

9. Singh NP, McCoy MT, Tice RR, Schneider EL. A simple technique for quantitation of low levels of DNA damage in individual cells. Exp Cell Res. 1988;175(1):184-91. https://doi. org/10.1016/0014-4827(88)90265-0

PMid: 3345800

10. Tsuda TM, Yoshikawa H, Shimuzu N, Takaoka K. Establishment of an osteoinductive murine osteosarcoma clonal cell line showing osteoblastic phenotypic traits. Bone. 1989;10:195-200. https://doi.org/10.1016/8756-3282(89)90053-7

11. Griffith D, Keck PC, Sampath TK, Rueger DC, Arlson WD. Three-dimensional structure of recombinant human osteogenic protein 1: Structural paradigm for the transforming growth factor $\beta$ superfamily. Proc Natl Acad Sci USA. 1996;93:878-83. https:// doi.org/10.1073/pnas.93.2.878

12. Wang RN, Green J, Wang Z, Deng Y, Qiao M, Peabody M, et al Bone morphogenetic protein (BMP) signaling in development and human diseases. Genes Dis. 2014;1(1):87-105. https://doi. org/10.1016/j.gendis.2014.07.005 